\title{
AN OPENEHR REPOSITORY BASED ON A NATIVE XML DATABASE
}

\author{
Linda Velte ${ }^{1}$, Tiago Pedrosa ${ }^{2}$, Carlos Costa $^{1}$ and José Luís Oliveira ${ }^{1}$ \\ ${ }^{1}$ University of Aveiro, DETI/EETA, Aveiro, Portugal \\ ${ }^{2}$ Polytechnic Institute of Bragança, 5301-854, Bragança, Portugal
}

\begin{abstract}
Keywords: EHR, openEHR, XML repository.
Abstract: $\quad$ openEHR is an open standard specification that describes the management, storage, retrieval and exchange of data in Electronic Health Record (EHR). Despite its growing importance in the field, the lack of open source solutions is hindering a larger visibility. In this paper we present an openEHR-based repository supported by a native XML database, which allows to store and query openEHR records through the DB service layer and a set of REST web services. The obtained results highlight the efficiency of this API and show that it can be used as a persistence component in any openEHR solution.
\end{abstract}

\section{INTRODUCTION}

An EHR allows an integrated access to the patients information as it gives the possibility to aggregate all medical data of the patient, and consequently, to have a patient centric storage approach. A patient's EHR must be accessible and the information understandable, no matter what hospital/medical institution he visits. To accomplish that, there must be an agreement on the language that is "spoken". This is done via standards that define not only how the information is structured and represented but also how it can be retrieved and shared between systems (Sunyaev, 2008). Existing standards can be divided into two major groups: 1) to define the content format, like, for instance, openEHR, MML (Medical Markup Language) and HL7 CDA (Health Level 7 Clinical Document Architecture) and 2) to define how the communication should be done like, for instance, WADO (Web Access to DICOM Persistent Objects), RID (Retrieve Information for Display) and XDS (Cross-Enterprise Document Sharing). Some standards can be included in both of these groups (DICOM Structured Reports and EHRcom) (MITRE, 2006). Within these standards, a great expectation is being put on openEHR, which usage has been increasing over the years (Eicheberg, 2005). Most standards focus on a specific area, such as DICOM focuses on digital imaging or HL7 on patients' administration. On other hand, openEHR has the goal to provide semantic interoperability between all medical specialties, reducing the needed of standards to a single one, in opposition to the several standards that are used nowadays.

An important aspect in openEHR solutions is the storage technology. This kind of repository needs a very generic database model, which is not supported by current implementations. Opereffa project (Opereffa, 2011) is an openEHR repository that uses a relational database, with just one table to store path/value pairs for each attribute. A strong drawback of this approach is that for patient centric queries the information has to be reassembled via complex joins operations.

In this paper we propose a repository supported by a XML database, where each patient has all his information in one record, simplifying storage and query procedures.

\section{OPENEHR}

OpenEHR is an open standard specification in health informatics that describes the management, storage, retrieval and exchange of data in EHR's (openEHR, 2011). In this standard, all data relative to a person is stored in a "one lifetime" vendor independent and centred EHR (Kalra, 2005).

The goal of openEHR standard is to achieve semantic interoperability regarding the whole health 
area. For this, a generic, dynamic health information model is needed, capable of storing new kinds of data without having to change the data model. The drawback of this kind of model is that there is no control about what information is stored, leading to low data quality and is not very different from an unstructured model. This issue was solved by an innovative modeling approach that creates a second level used to constrain the information (Bird, 2003). This decoupling methodology consists basically in the separation of information and knowledge. The result is a generic health record model that enables the storage of a wide variety of health information.

The openEHR architecture can be divided into two major parts: Reference Model the Archetype Model. The former allows interoperability because data is exchanged between systems only in terms of standard open reference model instances; the latter allows semantic interoperability. Additionally, there is a Service Model includes definitions of basic services in the health information environment, which are EHR centred.

The structure of the information is constrained by the archetype model, which defines exactly which data types, structures and values are valid, making the information both flexible and structured. An archetype represents a clinical concept and it is used to constrain instances of the openEHR information model by defining a valid structure, data types and values. An electronic health record that has been archetyped will have the same meaning no matter where it appears, allowing it to be shared by multiple health systems (Beale, 2002). Archetypes are defined using the Archetype Definition Language (ADL) (Beale and Heard 2005). To query openEHR information it was defined the Archetype Query Language (AQL) that allows to access the nodes in a similar way as a XPath query on an XML document (Beale, 2005).

There is already available a fully implemented version of the reference and archetype models - the openEHR Java Reference Implementation (Chen, 2007) - and many organizations are using or contributing to the openEHR standard. Two major references are Opereffa (Opereffa, 2011) and Kanolab (Kanolab, 2011).

\section{A OPENEHR REPOSITORY}

The purpose of our repository is to store electronic health records according to the openEHR standard, offering an API to perform database management functions (like insert, edit or delete a record) and to query the inserted records. To accomplish this task, a few aspects have to be considered. A very important one is the fact that the repository must store openEHR records using a dynamic database model. Another fact to have in consideration is that the repository is only a part of a healthcare system and, consequently, has to be inserted into this system architecture. To make this possible the implementation should have a well-defined set of services that are reusable by any application. Those services should provide the functionalities normally supported by a healthcare information system, namely operations related with records search and management.

The system architecture was designed to divide functionalities into three different layers, following the Service Oriented Architecture (SOA) model (Papazoglou, 2003). This approach has the advantage of providing reusable services, being possible to integrate implemented functionalities in any application. The result is shown in Figure 1.

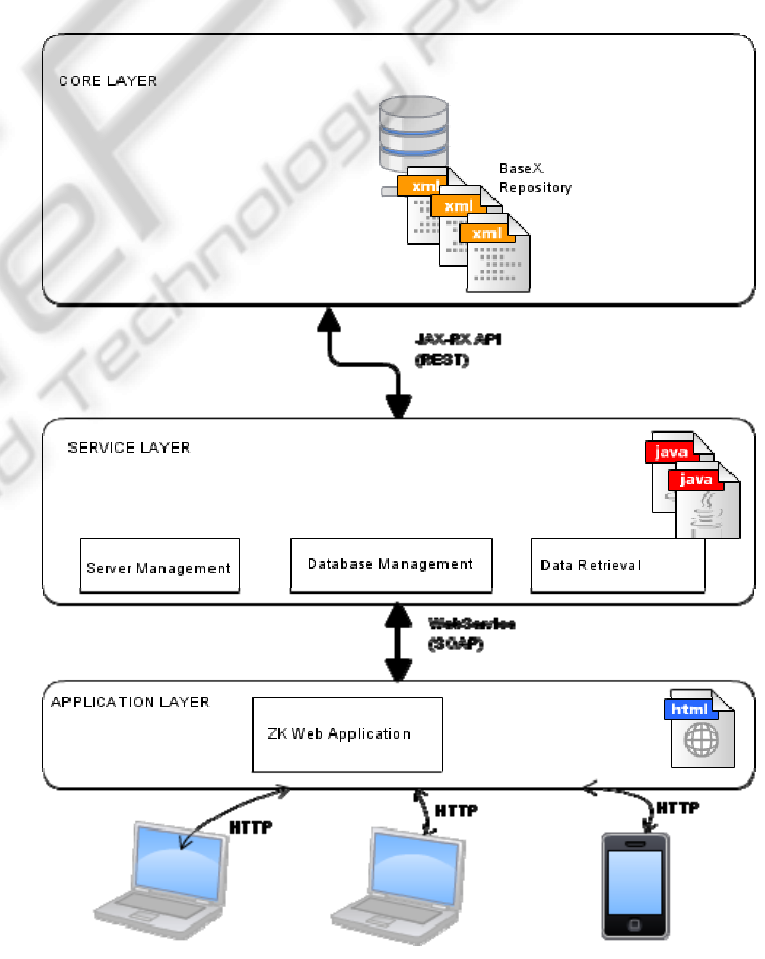

Figure 1: System Architecture.

\subsection{Core Layer}

The Core layer is responsible for managing the information to be stored. The storage technology used was a native XML database, a relatively new technology and, consequently an interesting case of study, namely to compare performance with 
relational approaches or with non-native XML databases (i.e. DBs that use a relation model with XML fields to store the data).

XML databases perform better when used in document centric applications, what makes it perfect to support a patient centric EHR repository. This kind of storage approach makes possible to have a XML file per patient containing all his medical data.

For our implementation we have used BaseX an open-source and platform-independent XML DB, written in Java, with an Xpath/XQuery processor, and with support for W3C Update and Full Text extensions. The $\mathrm{W} 3 \mathrm{C}$ Update is a great advantage regarding performance issues, allowing us to access to a specific position of the file to read or modify its value. Normally, other solutions obligate to read the entire file, change it and rewrite it. Moreover, it offers a RESTful API for accessing distributed XML resources, which provides a simple and fast access to databases though HTTP (using HTTP methods GET, PUT and DELETE).

The patient repository consists in one unique XML node that contains the EHR records, following the openEHR structure (Figure 2).

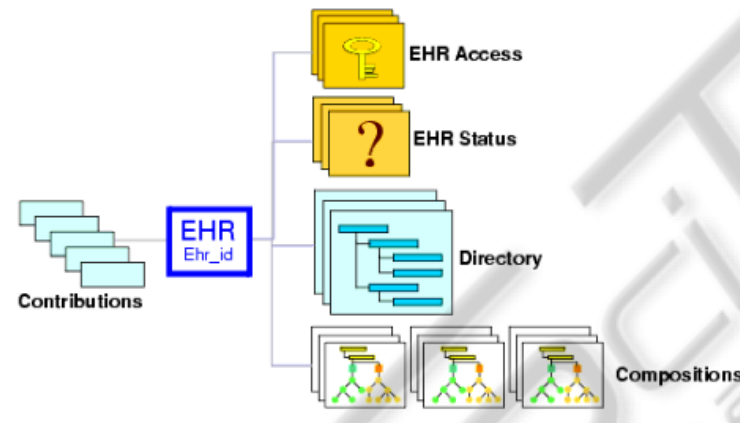

Figure 2: High Level Structure of the openEHR (openEHR 2011).

Each object is identified by an EHR ID and contains structured information that is versioned, plus a list of Contribution objects that act as audits for changes. The EHR Access and EHR Status objects contain control information, while the Directory (optional) can be used to hierarchically organize Compositions, objects that contain the patient medical information. Every EHR has also a list of Contributions that are used to save every change made to the record.

\subsection{Service Layer}

The set of implemented services allow the remote and transparent management of electronic health records storage and the query of information contained in these records. All the implemented services were implemented using SOAP and their functionalities can be divided into four groups according to their application area: DBManager, ServerManager, EHRManager and QueryService.

The DBManager module is responsible for the administrative operations on the repository, such as creating a new database or obtaining information about an already existing database. The EHRManager is responsible for all the Web Services related to record manipulation. This set of services cover the requisites regarding the information management of electronic health records, such as insertion, edition, or deletion of EHRs, compositions and contributions. The QueryService allows querying the data available in the repository. The two available services to retrieve information are executeQuery (free XPath/XQuery queries) and textQuery (full text search). The ServerManager file contains the services responsible for the server management, such as starting or stopping the BaseX server.

\subsection{Application Layer}

To validate the functionalities of the developed openEHR repository we have also developed a web application with a particular set of client services, namely: a) insert/edit/remove EHRs; b) view EHR content; c) get Composition list associated to an EHR; d) insert/edit/remove Compositions; e) query database (XPath and free text).

The application was developed in ZK, an opensource web solution written in Java that includes an Ajax-based event-driven engine and a rich set of components. The Web application works as an administration platform for any repository.

\section{EVALUATION}

To evaluate the performance of developed openEHR Repository systematic tests were made for series of 1000, 10000 and 30000 patient records. Several operations were analysed, included: a) Add a new EHR to the database; Search for a patient record; Search for an attribute; Add a composition to an existing record.

In our openEHR repository, the query processing is notably faster than the insertion process. Analysing the context where the repository will be used (health information systems), it is more important to have faster queries than to have faster insertions (add a new patient or add new data to the 
patient's record). After the tests we have concluded that while the query time for a record is independent of the volume of the database (approximately $0.65 \mathrm{~ms})$, the insertion time increases with the numbers of records already inserted (with 30000 records inserted, it took $423 \mathrm{~ms}$ to insert an additional one). The major conclusion is that this repository is best suitable for systems that require a fast query response to patient centric queries.

Using the index system mechanism offered by BaseX it was possible to obtain the same response time to query a patient's medical record, independently of the number of records inserted. It also made no difference which position the record occupies in the repository file.

Information centric queries, i.e. search for special attributes along a set of patients, are much slower and depend on the number of records inserted (Figure 3). In our approach, queries like, for instance, "how many patients have high blood pressure?" obligates to analyse the whole information inserted. To cope with this we need to deploy a more extensive indexing solution based on Lucene, for instance.

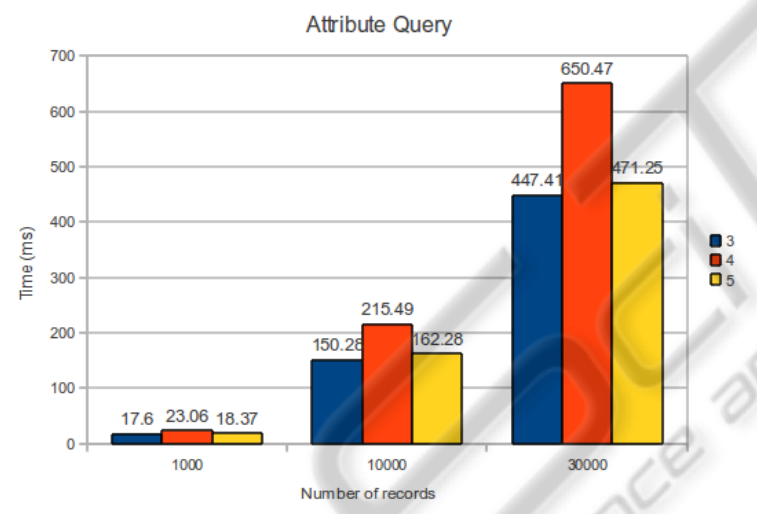

Figure 3: Attribute Search.

\section{CONCLUSIONS}

In this paper we have presented a generic and dynamic database, able to store electronic health records according to the openEHR standard. Additionally, the developed SOAP API offers a unified access to the repository, making it possible to integrate it with any kind of application. The goal is to promote the development of health information systems based on the openEHR standard, allowing an integration of all clinical areas (e.g. radiology, administration, pharmacy) and making the process more unified. Besides the repository and its clinical usage, we have also developed a set of web services and an administration web application that allow patients to enter their own medical information into secure health records. This support to PHR (Personal Health Records) systems is an important asset, considering the document centric approach of our solution - every patient has all his information stored in just one record.

The repository created and the services provided enable the creation of client applications in an easy way. The use of web services and the format of the record stored on XML allow the use of generic technology and programing languages.

\section{REFERENCES}

Beale T., 2002. Archetypes: Constraint-based domain models for future-proof information systems. OOPSLA 2002 workshop on behavioural semantics.

Beale, T., 2005. Archetype query language (aql), openEHR specification, openEHR foundation.

Beale, T., Heard, S., 2005. Archetype definition language (adl), openEHR specification, openEHR foundation.

Bird, L., Goodchild, A., Tun, Z., 2003. Experiences with a two-level modelling approach to electronic health records, Journal of Research and Practice in Information Technology, 35(2):121.

Chen, R., Klein, G., 2007. The openehr java reference implementation project. In Medinfo 2007, Proceedings of the 12th World Congress on Health (Medical) Informatics, Building Sustainable Health Systems, page 58. IOS Press.

Eichelberg, T. Aden, Riesmeier, J., Dogac, A., Laleci G.B., 2005. A survey and analysis of electronic healthcare record standards, ACM Computing Surveys (CSUR), 37(4):277-315.

Kalra, D., Beale, T., Heard, S, 2005. The openEHR foundation, Studies in Health Technology and Informatics, 115:153-173.

Kanolab, 2011. http://www.kanolab.info/index.php

Papazoglou, M. P., Georgakopoulos, D., 2003. Serviceoriented computing. Communications of the ACM, vol. 46 (10).

MITRE Coorporation, 2006. Electronic Health Records Overview. National Institutes of Health National, Center for Research Resources.

openEHR, 2011. http://www.openehr.org.

Opereffa, 2011, http://opereffa.chime.ucl.ac.uk/introducti on.jsf

Sunyaev, A., Leimeister, J. M., Schweiger, A., Krcmar, H., 2008. IT-Standards and Standardization Approaches in Healthcare. Encyclopedia of Healthcare Information Systems. 813-820. 